Un renouveau de l'enseignement des sciences

\title{
Réformer l'éducation scientifique : une prise de conscience mondiale.
}

Introduction

Florence Robine

\section{OpenEdition}

Journals

Édition électronique

URL : http://journals.openedition.org/ries/635

DOI : 10.4000/ries.635

ISSN : 2261-4265

Éditeur

Centre international d'études pédagogiques

Édition imprimée

Date de publication : 1 septembre 2009

Pagination : 27-34

ISSN : $1254-4590$

Référence électronique

Florence Robine, «Réformer l'éducation scientifique : une prise de conscience mondiale. », Revue internationale d'éducation de Sèvres [En ligne], 51 | septembre 2009, mis en ligne le 29 juin 2011, consulté le 19 avril 2019. URL : http://journals.openedition.org/ries/635 ; DOI : 10.4000/ries.635 


\section{Un renouveau de l'enseignement des sciences}

\section{Introduction}

\section{Réformer l'éducation scientifique: une prise de conscience mondiale}

\section{Florence Robine}

Après un numéro fort innovant sur l'enseignement des sciences humaines et sociales ${ }^{1}$, la Revue internationale d'Éducation de Sèvres a souhaité aborder un autre domaine important de connaissances, étudié dans tous les curricula du monde, celui des sciences. Ce thème a déjà fait l'objet d'un numéro de la Revue en juin 1997 ( $n^{\circ} 14$ ), sous le titre "l'éducation scientifique ». À l'époque, la réflexion est surtout centrée sur les nécessaires améliorations de la qualité de l'enseignement des disciplines scientifiques, jugé trop formel et trop sélectif. On voit se dessiner dans les articles d'alors les prémisses de projets pédagogiques innovants, axés sur une participation plus active des jeunes et sur un enseignement davantage orienté vers les aspects concrets, les implications sociétales, de la science.

Que s'est-il donc passé, pendant ces douze dernières années, qui justifie la rédaction d'un nouveau numéro consacré à l'enseignement des sciences? D'abord, et par-dessus tout, le développement sans précédent aux plus hauts niveaux politiques, dans pratiquement tous les pays du monde et dans les instances internationales, de l'attention portée à cette question. Appuyés sur de nombreux et récents rapports mettant en garde contre le déclin inquiétant des "vocations scientifiques", caractérisé par exemple par le recul relatif des diplômés en sciences de l'enseignement supérieur, les décideurs, souvent aiguillonnés par les milieux scientifiques ou économiques, se sont un peu partout penchés sur la question de l'éducation scientifique.

On le sait désormais, cette désaffection touche de manière différente les territoires, les champs disciplinaires et les niveaux d'enseignement, même si cela peut être interprété de façon variée, voire contesté par certains en arguant de modifications profondes et en cours du rapport des jeunes aux disciplines universitaires académiques, ou encore de structures évolutives du marché du

1. № 49, décembre 2008 "Quel avenir pour les études en sciences humaines ? ", coordonné par le professeur Michel Lussault, président du PRES Université de Lyon. 
travail. Cette idée a néanmoins généré une explosion impressionnante de rapports, d'études, d'abord de sociologues et de scientifiques, puis de milieux politiques dont témoigne l'abondante bibliographie réalisée ici par Bernadette Plumelle. Il est d'ailleurs intéressant de constater que, bien que les premiers signes soient déjà présents vers 1995 (à l'époque du numéro cité de la Revue par exemple), l'alerte n'est donnée que vers les années 2000 et après, engendrant alors une avalanche d'analyses et de recommandations. Tout cela se traduit aujourd'hui par de réelles mises en œuvre de nouvelles politiques éducatives, par l'impulsion de nouvelles pédagogies et réformes éducatives, dont témoignent les articles de ce numéro ; articles fort divers par leurs origines mais pour autant fort convergents dans les objectifs poursuivis et quelquefois même dans les modalités décrites.

\section{UN ENSEIGNEMENT EXEMPLAIRE DE LA MONDIALISATION DE L'ÉDUCATION}

\section{Un rapide historique}

La période des années quatre-vingt-dix, marquée par l'influence majeure d'une institution internationale, l'OCDE, a constitué un véritable tournant en matière de politique éducative. L'OCDE, prenant acte du passage des sociétés modernes à une ère "post-industrielle », s'empare de la notion de «capital humain » qui place au centre de la compétition économique mondiale la capacité des pays à forger une éducation de qualité, tout à la fois efficace et efficiente, apte à construire chez les individus les compétences essentielles pour que les pays développés continuent à faire bonne figure sur la scène internationale et préservent leurs capacités de développement économique et de paix sociale. Dès lors, parmi d'autres, les capacités à innover, à créer, les compétences d'ordre scientifique et technologique, les aptitudes de base en mathématiques sont affirmées comme essentielles face au défi de la mondialisation.

Plus intéressant, la mise en avant de ces compétences nouvelles s’inscrit également dans le nécessaire développement personnel des individus, et la construction d'une citoyenneté active désormais revendiquée.

Lors de la réunion du Conseil européen à Lisbonne, en mai 2000, l'Europe reprend à son compte l'essentiel de ces conceptions, et se donne alors l'objectif stratégique de «devenir l'économie de la connaissance la plus compétitive et la plus dynamique du monde, capable d'une croissance durable accompagnée d'une amélioration quantitative et qualitative de l'emploi et d'une plus grande cohésion sociale ", à l'horizon 2010. En 2005, le Conseil franchit un nouveau pas en adoptant cinq cibles prioritaires assorties d'indicateurs quantitatifs appelés "benchmarks", parmi lesquels l'engagement des jeunes dans les 
études scientifiques et le déséquilibre fille/garçon dans ces voies. La question de la "désaffection » est désormais portée politiquement au plus haut niveau européen, après les sonnettes d'alarme tirées par les divers rapports commandés par l'OCDE, le Global Science Forum, et la Commission européenne elle-même (rapport dit «Gago »), et elle est de plus clairement associée à celle de la qualité de l'éducation dans ce domaine.

Cette question n'intéresse pas que l'Europe, loin de là. Nous avons bien sûr mis en évidence l'implication de l'OCDE, mais on peut aussi noter l'intérêt des grandes nations des diverses régions du monde. Ainsi, aux ÉtatsUnis, le National Science Board publie-t-il en 2003 un rapport très critique où l'on peut lire que « la future force de la science américaine et de son ingénierie est en danger "; les États-Unis ne maintiennent leur taux de diplômés scientifiques que grâce à l'immigration. Là encore la sonnette d'alarme est tirée.

\section{Le rôle des évaluations et comparaisons internationales}

Un autre élément majeur est apparu sur la scène mondiale, dont les conséquences sur le pilotage éducatif - en particulier en ce qui concerne l'enseignement scientifique - sont visibles: l'émergence des évaluations internationales et des comparaisons, concernant les acquis des élèves, mesures d'une certaine qualité de l'éducation fournie. Pensons ainsi à TIMSS «trends in international mathematics and science study", mis en place depuis 1995 par l'IEA dans le but avoué de comparer les résultats des élèves américains avec ceux des autres pays, et à PISA "Program for International Student Assessment » ou programme international pour le suivi des acquis des élèves, réalisé tous les trois ans depuis 2000 sous l'égide de l'OCDE.

Au regard de la mondialisation - voire la globalisation - de l'éducation, l'évaluation PISA est tout à fait exemplaire. Son développement fulgurant, sa prise en compte grandissante dans l'évaluation et donc le pilotage des politiques publiques est emblématique. En ce qui concerne le sujet spécifique de l'enseignement des sciences, ces évaluations ont eu des effets en deux temps, assez si ce n'est totalement contradictoires. Wynne Harlen le décrit bien dans son article consacré à la Grande Bretagne; les comparaisons internationales ou nationales des acquis des élèves ont d'abord provoqué presque partout un repli vers les enseignements fondamentaux (maitrise de l'écrit, compétences de base en mathématiques) au détriment des sciences et autres activités considérées comme accessoires. Le choc en retour des évaluations dans le domaine scientifique a pu provoquer par la suite une réorientation des stratégies. De plus, les objectifs des évaluations en science, davantage centrées sur des compétences innovantes que les syllabi classiques, ont posé clairement la question des contenus de l'enseignement des sciences et de sa pédagogie. 


\section{Les Politiques Éducatives MISES EN GUVRE}

Les articles de ce numéro le montrent: la prise de conscience de l'acuité de la question a suscité la mise en ouvre de politiques éducatives qui montrent à l'évidence des similitudes dans leurs objectifs, voire dans leurs stratégies.

\section{Des objectifs identifiés, multiples, quelquefois concurrents}

On note avec intérêt, dans la lignée des recommandations et travaux réalisés par les grandes instances internationales, l'émergence quasi universelle de la contribution de l'enseignement scientifique à la formation du nouveau citoyen du XXI ${ }^{\mathrm{e}}$ siècle. Y. Wei, dans l'article consacré à la Chine, parle ainsi du "développement social durable»; W. Harlen, pour le cas de la GrandeBretagne, écrit un plaidoyer pour la «littéracie scientifique » du citoyen; les auteurs suédois rappellent combien les mathématiques constituent une compétence-clé pour le citoyen moderne; l'article indien montre combien la construction de valeurs est au premier plan des réformes curriculaires, y compris en sciences. L'un des objectifs essentiels, rappelle Pierre Léna, est bien de lutter contre l'isolement de la science et développer partout le goût de la culture qu'elle délivre, part essentielle du patrimoine commun moderne.

Parallèlement, s'affirme la nécessité d'une réelle démocratisation de l'enseignement des sciences, c'est-à-dire d'un développement d'une éducation de qualité pour tous. A. Sané, prenant appui sur le cas du Sénégal, rappelle l'impérieuse nécessité pour les pays d'Afrique de travailler sur les questions "d'équité et de justice démocratique ", en gommant les profondes disparités de territoires, de ressources qui grèvent le processus d'apprentissage des sciences pour tous. S. Jokic, pour la Serbie, montre comment ce pays, qui avait jusquelà plutôt concentré ses efforts de développement et de rénovation de l'enseignement des sciences aux étudiants dits doués, s'attelle désormais à une éducation modernisée pour tous.

Cela ne saurait faire oublier cependant l'autre versant des politiques de rénovation entreprises, suite logique des éléments de constat présentés, destiné à promouvoir l'implication des jeunes dans les études et carrières scientifiques et technologiques et à accroître le niveau de formation dans ces domaines, de manière à pouvoir soutenir la concurrence internationale dans ce contexte effréné de globalisation. De nombreux auteurs de nos articles l'indiquent: il s'agit bien aussi de soutenir l'orientation professionnelle des jeunes, et pour cela d'analyser la part que doit prendre l'enseignement des sciences dans cette mission, au-delà des questions sociétales et économiques qui pèsent évidemment aussi sur cette problématique. 
À bien y regarder, il s'agit là d'objectifs fort louables mais dont la convergence n'est pas assurée; au moins nécessite-t-elle que l'on prenne ces questions à bras le corps pour tenter de voir comment l'on peut concilier l'éducation scientifique pour tous et la formation de pointe pour les futurs scientifiques.

\section{Des démarches et stratégies communes partout affirmées}

Pour tenter d'atteindre les objectifs fixés, il est frappant de constater l'homogénéité des démarches et des stratégies poursuivies par les États. J'en caractériserai ici les traits principaux qui semblent se dégager.

Tout d'abord, l'accent très général mis sur l'enseignement primaire. Tous les textes internationaux l'affirment, nos auteurs le confirment : "sooner is better ", le plus tôt est le mieux. C'est évidemment le credo de l'opération $L a$ main à la pâte, dont l'un des fondateurs, Pierre Léna, nous rappelle la genèse et le développement, thème repris par les articles chinois, serbe et britannique. C'est aussi le propos général de l'étude de cas indienne.

Également: l'importance accordée par tous les pays à la qualité de la formation et du développement professionnel des enseignants. Cet élément est considéré par tous nos auteurs comme un point clé des rénovations en cours : soit qu'il s'agisse de former des enseignants, en général du primaire, qui n’ont reçu qu'une éducation scientifique initiale de faible niveau (en Chine, en France, en Grande-Bretagne, en Serbie), soit qu'il s'agisse d'accentuer la formation des enseignants spécialisés dans les disciplines scientifiques (en Suède, au Sénégal), de la façon de faire évoluer leurs pratiques pédagogiques et de mettre à leur disposition des ressources pédagogiques innovantes et motivantes. À cet égard, l'article présenté ici par un groupe de recherche impliqué dans un projet européen s'applique à montrer les obstacles à surmonter pour construire une dynamique de formation efficace, construite sur des objectifs identifiés et néanmoins adaptée aux contextes nationaux spécifique ; l'article indien attire, lui, notre attention sur les différentes stratégies de formation à mettre en ouvre et leur inégale contribution au changement de pratique des enseignants.

L'un des traits saillants des stratégies mises en place est l'aspect central de la coopération internationale. Cette caractéristique originale est un des points remarquables de l'enseignement des sciences et participe de cet aspect frappant de "mondialisation" déjà souligné. Evidemment, l'universalité des contenus et des concepts enseignés, l'avènement d'un monde dominé par la technologie permettent de comprendre sur quel terreau commun cette coopération internationale peut s'installer. Qu'elle soit aussi centrale reste néanmoins un élément majeur. L'article portant sur le projet européen en fait évidemment l'un de ses thèmes principaux, que nous retrouvons sans exception dans tous les textes de ce dossier. 
Et finalement, l'importance et la convergence des réformes pédagogiques et didactiques apparaissent comme l'un des enseignements essentiels de ce numéro 51 de la Revue : partout s'est imposée une vision renouvelée de la pédagogie des sciences, basée sur des démarches actives d'investigation, de résolution de problèmes, sur le développement de compétences en lien avec les questions actuelles et les problèmes sociétaux. Prônée désormais par les textes internationaux (évaluation PISA, rapports de l'OCDE et de la Commission Européenne), elle est reprise partout et souvent centrale dans les actions de formation des enseignants et dans les développements curriculaires.

\section{LA DÉMARCHE D'INVESTIGATION : UNE RÉPONSE UNIVERSELLE?}

La lecture de tous les articles le montre : l'IBSE (Inquiry Base Science Education) et le PBL (Problem Based Learning), cette stratégie pédagogique fortement liée aux démarches constructivistes (Inde, Chine, article européen) répond assez clairement aux objectifs visés, en particulier de développement d'une nouvelle citoyenneté éveillée, active, munie de compétences socioémotionnelles qui favorisent une vraie insertion positive dans le monde d'aujourd'hui. Elle permet aussi une meilleure compréhension de ce qu'est actuellement l'activité scientifique, basée sur la curiosité, l'inventivité, la recherche plutôt que sur la répétition de savoirs décontextualisés. C'est bien dans cette perspective que s'est élaborée la démarche type La main à la pâte, dont le succès international montre à l'évidence qu'elle répond à des aspirations actuelles, ou bien dans les nouvelles pédagogies mises en place dans l'enseignement des mathématiques (Suède). Pour autant, cette unanimité interroge et mérite que l'on se penche davantage sur certains points.

\section{Quelle effectivité concernant la mise en œuvre ?}

Au-delà des intentions avouées et des cadres mis en place, la question de l'effectivité des réformes mises en œuvre se pose. Pierre Léna le rappelle, celle-ci se heurte sur le terrain à de nombreux écueils. Le cas de la France est à cet égard intéressant : la démarche instituée nationalement en 2002, et pilotée par le ministère, après l'expérimentation La main à la pâte dans un certain nombre d'écoles, a certes permis un bond dans l'imprégnation scientifique des élèves à l'école primaire, mais peine encore à dépasser la barre des 30 à $40 \%$ d'écoles impliquées. De nombreux articles vont dans le même sens (Grande-Bretagne, Serbie). Tous le rappellent : il est nécessaire de tout à la fois mettre en place un plan volontariste piloté au plus haut niveau (Suède, Chine, Sénégal) et de maintenir un effort au long cours, ancré dans une vraie volonté politique. L'article indien met de plus l'accent sur le fait que cette rénovation pédagogique ne peut être conçue en dehors d'une stratégie holistique qui s'attaque à tous les aspects de l'enseignement. 


\section{Quelles bases théoriques?}

Au-delà des impressions, de la sensation profonde que ces stratégies actives sont plus en phase avec les attentes des jeunes, avec leur rapport aux savoirs, et plus à même de les attirer vers les sciences, il convient de se demander si l'on peut asseoir ces réformes didactiques et pédagogiques sur des éléments conceptuels plus solides. Il s'agit bien de prendre un peu de recul par rapport à "l'air du temps", au dernier engouement dans lequel tout le monde s'engouffre sans autre véritable argument que le fait que le monde entier s'y engage, même si ce pouvoir de conviction force le respect. L'article de $\mathrm{M}^{\mathrm{me}}$ Wei a le grand mérite d'affronter cette question difficile. La Chine, consciente du fossé installé entre les pédagogues et la recherche en sciences de l'éducation ainsi qu'en neurosciences et sciences cognitives, s'est adjoint le concours de ces domaines dans le développement même du programme de réformes de l'enseignement des sciences. Il s'agit bien ici d'interroger ce qui semble partout ailleurs admis : pourquoi doit-on commencer cette pédagogie dès l'enfance ? En quoi des démarches pédagogiques actives, basées sur l'investigation, sont elles préférables ? Quels bénéfices quant au développement de nouvelles compétences, de type relationnel, langagier ou social ? L'article indien interroge ainsi fort directement les présupposés des stratégies constructivistes, des pédagogies actives basées sur la découverte et l'expérience. Le fait même de s'autoriser de tels questionnements, de réunir pédagogues, décideurs et chercheurs pour y réfléchir et en discuter, constitue un élément clé du renouveau de cet enseignement, tel que le décrit l'article chinois. La même logique est à l'œuvre dans l'exposé réalisé par le groupe de recherche interuniversitaire européen, cette fois sur le champ de la formation des maîtres. On voit là naître un nouveau pilotage, décomplexé, audacieux, de réformes curriculaires qui, à n'en pas douter, intéressera nos lecteurs de par le monde.

\section{Quels résultats?}

On aborde ici l'une des questions les plus délicates, toujours présentes lorsque l'on étudie la mise en place de telles rénovations. Peut-on évaluer les résultats des politiques mises en place, à l'aune des objectifs fixés ?

Les efforts considérables consentis en termes de formation nouvelles des professeurs, de mise à disposition de structures et ressources nouvelles sontils suivis d'effets? Les élèves apprennent-ils mieux les sciences? Les aiment-ils, les comprennent-ils mieux en tant que citoyens avertis ? Est-ce positif en termes d'orientation vers les métiers scientifiques et technologiques?

Pierre Léna rappelle combien cette question, certes importante, trouve difficilement de réponse. De plus, ne l'oublions pas, l'appétence envers les sciences, leur image et retentissement dans une société tiennent à de nombreux facteurs sur lesquels l'école n'a que peu d'influence. Cela ne dédouane en rien 
son rôle, car on sait combien les enquêtes internationales ont partout pointé l'influence de l'enseignement des sciences, son côté académique, déconnecté des réalités du monde, sur le désintérêt des jeunes. À cet égard, les quelques tentatives louables d'évaluation de cet aspect ont souvent montré que les démarches d'investigation, les pédagogies actives, si elles n'amélioraient pas de manière significative les connaissances des élèves en sciences, leur permettaient d'en développer une image plus valorisante ainsi que des compétences personnelles (autonomie, prise d'initiative, travail en groupe) plus affirmées. Le champ de l'évaluation est, à n'en pas douter, un chantier encore largement ouvert.

\section{Quelles autres pistes à explorer?}

Cette réforme de la pédagogie des sciences peut-elle à elle seule inverser le cours des évènements et modifier la perception qu'ont les jeunes des activités scientifiques, à travers ce qu'ils en voient et pratiquent à l'école? La question posée renvoie à bon nombre de points que nous venons de soulever, mais elle interroge aussi sur les voies alternatives à explorer. La belle unanimité des articles publiés ici montre que nous n'en sommes pas encore là, et que pour l'instant les États et instances internationales ont jeté l'essentiel de leur force sur une rénovation des méthodes et des ressources d'enseignement, dont on voit en effet l'urgence (Sénégal). Reste encore à s'interroger, à mon sens, sur le contenu des enseignements, sur les savoirs à transmettre et à construire, en phase avec les objectifs posés visant tout à la fois au développement d'une citoyenneté éclairée et à l'appréhension intelligente d'un monde scientifiquement et technologiquement aussi avancé. Les problématiques mondiales auxquelles les jeunes vont devoir faire face dans les années qui viennent (défi énergétique, alimentation et ressources en eau, questions de santé, etc.) requièrent à l'évidence des savoirs plus complexes et davantage décloisonnés que ceux que nous enseignons encore majoritairement dans nos écoles, dans nos lycées et dans nos universités. La formidable, mais redoutable puissance de la science entraîne de nouvelles responsabilités, d'ordre éthique, social, politique. La montée des obscurantismes, l'affrontement grandissant entre savoirs et croyances, nécessite à l'évidence une vraie dimension épistémologique à l'enseignement scientifique et plus encore à la formation des maîtres, ainsi qu'une inscription dans l'histoire des idées et dans l'histoire tout court. Nous espérons que ce numéro, au cœur de l'une des questions pédagogiques, et finalement sociétales, les plus vives, fort de la diversité et de l'acuité des études de cas présentées, sera riche de réflexions et d'idées novatrices pour chacun de nos lecteurs. 